\section{Are Tight Glycemic Targets Achieved Through Intensive Insulin Infusion Still Applicable in the Intensive Care Unit?}

\section{THE "PRO" SIDE}

Clinical trials have shown that over $98 \%$ of patients in the intensive care unit (ICU) require insulin therapy to maintain normoglycemia. ${ }^{1-4}$ Hyperglycemia is associated with higher morbidity and mortality for patients with traumatic brain injuries, ${ }^{5-7}$ sepsis, ${ }^{8}$ hematologic malignancy, ${ }^{9}$ and nondiabetic stroke. ${ }^{10}$ Among those who have had myocardial infarction, hypoglycemia is associated with an increased risk of congestive heart failure and cardiogenic shock. ${ }^{11}$ However, the use of intensive insulin therapy (IIT) to manage hyperglycemia in the ICU is a controversial topic. Although some studies have shown that IIT improves outcomes, recent trials have questioned the benefit of IIT in light of the potential for increased frequency of hypoglycemia. The question to be addressed here is: Does implementing IIT for all ICU patients improve outcomes? Various trials will be discussed to illustrate that treatment of hyperglycemia with IIT does improve outcomes.

Van den Berghe and others conducted both of the landmark trials supporting IIT in the ICU setting. ${ }^{1,2}$ Their first trial involved patients in a surgical ICU. ${ }^{1}$ Participants were randomly assigned to IIT (blood glucose target 4.4-6.1 mmol/L) or conventional treatment (blood glucose target 10-11.1 mmol/L). The study was terminated early after a planned interim analysis indicated significantly lower ICU mortality ( $4.6 \%$ versus $8.0 \%$, $p<0.04)$ and in-hospital mortality $(7.2 \%$ versus $10.9 \%, p=$ $0.01)$ in the IIT group. The mortality benefit was most evident among patients requiring intensive care for longer than 5 days. The greatest reduction in mortality involved deaths due to multisystem organ failure, followed by deaths due to severe brain damage. ${ }^{1}$ Other benefits included reductions in blood stream infections, critical illness polyneuropathy, ${ }^{12}$ blood transfusions, ${ }^{1}$ acute renal failure, ${ }^{2}$ and duration of mechanical ventilation. ${ }^{13}$ These authors subsequently conducted a similar study enrolling medical ICU patients. ${ }^{2}$ Among patients who stayed in the ICU for at least 3 days, in-hospital mortality was significantly lower in the IIT group than in the group receiving conventional therapy ( $43.0 \%$ versus $52.5 \%, p=0.009)$. The results of these studies suggest that IIT has particular benefits for those with a prolonged stay in the ICU.

Recent studies that have conflicted with the findings summarized above are the Normoglycaemia in Intensive Care Evaluation - Survival Using Glucose Algorithm Regulation (NICE-SUGAR) study, ${ }^{4}$ a prospective randomized multicentre controlled trial of tight glucose control by IIT in adult ICUs (the Glucontrol study), ${ }^{14}$ and a trial of IIT and pentastarch resuscitation in severe sepsis (VISEP, the Efficacy of Volume Substitution and Insulin Therapy in Severe Sepsis study). ${ }^{3}$ The NICE-SUGAR study ${ }^{4}$ compared IIT (target 4.5-6.0 mmol/L) with conventional glucose control (target $\leq 10 \mathrm{mmol} / \mathrm{L}$ ). The mortality rate was significantly greater among participants who were randomly assigned to receive IIT than among control patients $(27.5 \%$ versus $24.9 \%, p=0.02)$, with no difference in outcomes between surgical or medical patients. However, a subgroup analysis revealed possible trends of benefit with IIT among patients who had experienced trauma $(p=0.07)$ and those receiving corticosteroids $(p=0.06)$. The Glucontrol study compared IIT (target $4.4-6.1 \mathrm{mmol} / \mathrm{L}$ ) with intermediate glucose control (target $7.8-10.0 \mathrm{mmol} / \mathrm{L}$ ) in medicosurgical ICU patients. There was no difference in mortality between the 2 groups. ${ }^{14}$ The VISEP investigators compared IIT (target 4.4-6.1 $\mathrm{mmol} / \mathrm{L}$ ) with conventional insulin therapy (target $10-11.1 \mathrm{mmol} / \mathrm{L}$ ) in patients with severe sepsis. At 28 days, there was no significant difference between the 2 groups in the rate of death $(p=0.74)$ or the mean score for organ failure $(p=0.88)$. As for concerns about hypoglycemia and its proposed harmful effects, no long-term sequelae were reported in the NICE-SUGAR study, ${ }^{4}$ and this was not considered to be a safety concern in the Glucontrol study. ${ }^{14}$ In VISEP, the IIT arm was terminated early because of an increased number of hypoglycemic events, which were not found to result directly in death. ${ }^{3}$

The studies described above had limitations. The NICE-SUGAR study used a subjective inclusion criterion (expected length of stay in the ICU), and the glucose concentration in the IIT group was modestly above the target range. Furthermore, $10 \%$ of patients in the IIT group discontinued the study treatment prematurely. Given that this analysis was an intention-to-treat analysis, the extent to which these patients contributed to the difference in mortality between the 2 groups remains unclear. ${ }^{4,15}$ The Glucontrol study was stopped early because of high rates of protocol violations and was therefore underpowered. Furthermore, variations in the number of patients recruited at each centre, differences in experience with glucose control within the ICU team, therapeutic limitations, and/or prolongations of ICU stays for nonmedical reasons might have represented confounding factors. The pumps used for insulin infusion were not standardized, leading to a possibility of inaccuracies in the rate of infusion. Potential reasons for hypoglycemia, such as discontinuation or lowering of the infusion rate of enteral or parenteral nutrition solutions, were not recorded. Furthermore, sampling of capillary blood for blood glucose was an option, even though this method is sometimes inaccurate in the ICU setting because it may overestimate the reference standards and potentially miss hypoglycemic episodes. ${ }^{16}$ In the VISEP study, hypoglycemia was shown to be an independent risk factor for death from any cause; however, the severity of clinical sequelae from hypoglycemic events was unknown. 
Interestingly, although IIT resulted in more hypoglycemic events in the VISEP study $(p<0.001)$, IIT was not a risk factor for death $(p=0.72)$. Therefore, hypoglycemia may be a marker of poor outcome, independent of insulin therapy in patients with sepsis. ${ }^{3}$

In 2009 Griesdale and others ${ }^{17}$ published a meta-analysis involving over 13500 critically ill patients. The pooled relative risk (RR) of death with IIT (target $\leq 6.1 \mathrm{mmol} / \mathrm{L}$ ) compared with conventional management (target $\leq 8.3 \mathrm{mmol} / \mathrm{L}$ ) was 0.93 (95\% confidence interval [CI] 0.83-1.74). However, there was a mortality benefit for surgical ICU patients (RR 0.63, 95\% CI $0.44-0.91)$. The patients included in this analysis had different severities of illness and event rates, and the ICU settings had different management and nutritional strategies. The authors were therefore unable to exclude the possibility that some patients might benefit from IIT. ${ }^{17,18}$

Perhaps the main concern regarding tight glycemic control in the ICU setting ought to be shifted from concerns about hypoglycemia to the clinical consequences of fluctuations in blood glucose. It remains unclear whether the association between hypoglycemia and mortality in IIT is coincidental or causal. The clinical relevance of brief hypoglycemic episodes, independent of the severity of illness, is poorly defined. In the studies performed by Van den Berghe and collaborators, hypoglycemic events in the IIT group had no serious complications. ${ }^{1,2}$ In the Glucontrol study, hypoglycemia due to IIT was not a safety concern of the Data and Safety Monitoring Board when it halted the study. ${ }^{14}$ In the NICE-SUGAR study, the rate of hypoglycemia among patients with IIT was lower than in the trials discussed here, and no long-term sequelae of severe hypoglycemia were reported. ${ }^{4}$ Conversely, fluctuations in blood glucose do deserve our attention. In the Glucontrol study, more than $50 \%$ of blood glucose readings for patients in the IIT group were out of range. ${ }^{14} \mathrm{It}$ is this variability in blood glucose level that is thought to be a strong independent predictor of death in the ICU. ${ }^{3,16}$ Perhaps the lack of benefit and/or higher mortality rate may also be attributed to glucose variability, which occurs secondary to poor adherence with the insulin protocol and inaccuracies in glucose monitoring. ${ }^{19}$

It may be true that IIT is not suitable for all ICU patients, but haphazard glycemic control should not be ignored. Striving for moderately tight glycemic goals to avoid hypoglycemia, with minimal variability in glucose, strikes a safer balance. Unfortunately, the available evidence does not allow definitive recommendations for optimal targets. The clinical significance of episodic hypoglycemia occurring in association with IIT needs to be evaluated within the context of glucose variability. As illustrated by the Van den Berghe studies, ${ }^{1,2}$ benefits are realized when IIT targets are reached through strict adherence to insulin protocols. It may very well be that no benefits are apparent when insulin protocols are violated and glucose levels fluctuate, as was observed in the Glucontrol study. Overall, it seems that IIT confers morbidity and mortality benefits when implemented in specific ICU populations within the context of well-defined IIT protocols.
References

1. Van den Berghe G, Wouters P, Weekers F, Verwaest C, Bruyninckx F, Schetz $\mathrm{M}$, et al. Intensive insulin therapy in the critically ill patients. $N$ Engl J Med 2001;345(19):1359-1367.

2. Van den Berghe G, Wilmer A, Hermans G, Meersseman W, Wouters PJ, Milants I, et al. Intensive insulin therapy in the medical ICU. $N$ Engl J Med 2006;354(5):449-461.

3. Brunkhorst FM, Engel C, Bloos F, Meier-Hellman A, Ragaller M, Weiler $\mathrm{N}$, et al. Intensive insulin therapy and pentastarch resuscitation in severe sepsis. N Engl J Med 2008;358(2):125-139.

4. NICE-SUGAR Study Investigators; Finfer S, Chittock DR, Su SY, Blair $\mathrm{D}$, Foster D, Dhingra V, et al. Intensive versus conventional glucose control in critically ill patients. N Engl J Med 2009;360(13):1283-1297.

5. Krinsley JS. Association between hyperglycemia and increased hospital mortality in a heterogeneous population of critically ill patients. Mayo Clin Proc 2003;78(12):1471-1478.

6. Yendamuri S, Fulda GJ, Tinkoff GH. Admission hyperglycemia as a prognostic indicator in trauma. J Trauma 2003;55(1):33-38.

7. Rovlias A, Kotsou S. The influence of hyperglycemia on neurological outcome in patients with severe head injury. Neurosurgery 2000; 46(2):335-342.

8. Leonidou L, Mouzaki A, Michalaki M, DeLastic AL, Kyriazopoulou V, Bassaris HP, et al. Cytokine production and hospital mortality in patients with sepsis-induced stress hyperglycemia. I Infect 2007;55(4): 340-346.

9. Ali NA, O’Brien JM Jr, Blum W, Byrd JC, Klisovic RB, Marcucci G, et al. Hyperglycemia in patients with acute myeloid leukemia is associated with increased hospital mortality. Cancer 2007;110(1):96-102.

10. Capes SE, Hunt D, Malmberg K, Pathak P, Gerstein HC. Stress hyperglycemia and prognosis of stroke in nondiabetic and diabetic patients: a systematic overview. Stroke 2001;32(1):2426-2432.

11. Capes SE, Hunt D, Malmberg K, Gerstein HC. Stress hyperglycaemia and increased risk of death after myocardial infarction in patients with and without diabetes: a systematic overview. Lancet 2000;355(9206):773-778.

12. Van den Berghe G, Schoonheydt K, Becx P, Bruyninckx F, Wouters PJ. Insulin therapy protects the central and peripheral nervous system of intensive care patients. Neurology 2005;64(8):1348-1353.

13. Hermans G, Wilmer A, Meersseman W, Milants I, Wouters PJ, Bobbaers $\mathrm{H}$, et al. Impact of intensive insulin therapy on neuromuscular complications and ventilator dependency in the medical intensive care unit. Am J Respir Crit Care Med 2007;175(5):480-489.

14. Preiser JC, Devos P, Ruiz-Santana S, Mélot C, Annane D, Groeneveld J, et al. A prospective randomised multi-centre controlled trial on tight glucose control by intensive insulin therapy in adult intensive care units: the Glucontrol study. Intensive Care Med 2009;35(10):1738-1748.

15. Inzucchi SE, Siegal M. Glucose control in ICU-how tight is too tight? $N$ Engl J Med 2009;360(13):1346-1349.

16. Egi M, Bellomo R, Stachowski E, French CJ, Hart G. Variability of blood glucose concentration and short-term mortality in critically ill patients. Anesthesiology 2006;105(2):244-252.

17. Griesdale D, de Souza R, van Dam RM, Heyland DK, Cook DJ, Malhotra A, et al. Intensive insulin therapy and mortality among critically ill patients: a meta-analysis including NICE-SUGAR study data. CMAJ 2009;180(8):821-827.

18. Van den Berghe G, Mesotten D, Vanhorebeek I. Intensive insulin therapy in the intensive care unit. CMAJ 2009;180(8):799-800

19. Fahy BG, Sheehy AM, Coursin DB. Glucose control in the intensive care unit. Crit Care Med 2009;37(5):1769-1776.

\section{An Sada, BScPhm, PharmD}

Guelph, Ontario

Karen Wai Kwan Ng, BScPhm, ACPR

PharmD student

Lesley Dan Faculty of Pharmacy

University of Toronto

Toronto, Ontario 


\section{THE "CON" SIDE}

During critical illness, glucose homeostasis is disrupted, which often results in hyperglycemia. Observational studies have revealed an association between uncontrolled hyperglycemia and poor clinical outcomes in critically ill patients with various conditions. ${ }^{1-4}$ In 2001, Van den Berghe and others ${ }^{5}$ reported that maintenance of normoglycemia (target 4.4-6.1 $\mathrm{mmol} / \mathrm{L}$ ) with intensive insulin therapy (IIT) for patients in the surgical intensive care unit (ICU) was associated with a 32\% lower risk of in-hospital mortality. That pivotal trial persuaded many institutions to adopt IIT. Their enthusiasm was understandable: maintenance of normoglycemia makes sense intuitively, and IIT seems to be an inexpensive way to improve the outcomes of critically ill patients. However, in subsequent trials, the mortality benefits of IIT could not be confirmed in other ICU patients. ${ }^{6-9}$ Therefore, tight glycemic control (target 4.4-6.1 mmol/L) with IIT should not be applied in medical or mixed (medical and surgical) ICU settings.

In their single-centre, prospective, open-label, randomized controlled trial involving 1548 critically ill patients who had undergone surgery, Van den Berghe and others 5 found that, compared with a conventional blood glucose target (10-11.1 $\mathrm{mmol} / \mathrm{L}$ ), tight glycemic control (target 4.4-6.1 $\mathrm{mmol} / \mathrm{L}$ ) achieved through IIT was associated with lower ICU mortality (8.0\% versus 4.6\%; absolute risk reduction [ARR] 3.4\%, number needed to treat [NNT] 29) and lower in-hospital mortality (10.9\% versus 7.2\%; ARR 3.7\%, NNT 27). Severe hypoglycemia (blood glucose $\leq 2.2 \mathrm{mmol} / \mathrm{L}$ ) was significantly higher in the IIT group $(5.1 \%$ versus $0.8 \%$, absolute risk increase [ARI] 4.3\%, number needed to harm [NNH] 23). ${ }^{5}$ The investigators claimed that the severe hypoglycemia was not detrimental, ${ }^{5}$ but others have expressed concern that neurologic and cardiac sequelae may outweigh the benefits of tight glycemic control. ${ }^{10,11}$

Despite its compelling results, the study by Van den Berghe and others ${ }^{5}$ had several limitations. It included predominantly ICU patients who were receiving mechanical ventilation after cardiac surgery and who had low Acute Physiology and Chronic Health Evaluation II (APACHE II) scores (mean score 9). Therefore, the results cannot be extrapolated to other groups of ICU patients or to individuals with higher risk of death. Importantly, the trial was stopped early for efficacy reasons; hence, it may have overestimated treatment effects but lacked the ability to detect harm. ${ }^{12-14}$ Furthermore, routine IV administration of glucose (200-300 g/24 h) and frequent use of total parenteral nutrition in the study did not reflect current practice. ${ }^{15-17}$ One might speculate that IIT led to a mortality benefit by offsetting the deleterious effect of "excessive" parenteral glucose. ${ }^{18}$ Finally, glucose concentrations were determined from whole blood by means of point-of-care arterial blood gas analyzers. However, point-of-care systems, including "bench-top" analyzers and glucometers, are unreliable for determining low-range blood glucose values, and hypoglycemia may therefore have been underreported. ${ }^{19}$

In 2006, Van den Berghe and others ${ }^{6}$ repeated their study in a medical ICU setting. Because the participants in the later study had higher APACHE II scores, a similar or greater mortality benefit was expected. Although there were differences in morbidity for the IIT group (lower incidence of newly acquired kidney injury and earlier weaning from mechanical ventilation), an intention-to-treat analysis of data from 1200 patients revealed no statistically significant difference in hospital mortality (IIT $37.3 \%$ versus control $40.0 \%, p=0.33$ ). A post hoc analysis showed a mortality benefit for the subgroup of patients with a length of stay of 3 days or longer (IIT $43.0 \%$ versus control $52.5 \%, p=0.009) .{ }^{6}$ In clinical practice, selecting newly admitted ICU patients who may benefit from IIT is difficult. Importantly, in both of the studies by Van den Berghe's group, insulin adjustments were made at the bedside by dedicated "insulin teams" who followed a general dosing algorithm; such a practice may not be feasible in most facilities. ${ }^{5,6}$

Three subsequent studies $^{7-9}$ have shown no mortality or morbidity benefits with tight glycemic control, which has raised concerns about the safety and efficacy of this approach. The Efficacy of Volume Substitution and Insulin Therapy in Severe Sepsis (VISEP) study ${ }^{7}$ was a multicentre randomized controlled trial comparing a conventional blood glucose target (10-11.1 $\mathrm{mmol} / \mathrm{L})$ with an intensive target (4.4-6.1 $\mathrm{mmol} / \mathrm{L})$. VISEP showed no statistically significant difference in 28-day mortality (IIT $24.7 \%$ versus control $26 \%, p=0.74$ ) or morbidity (defined as ventilator-free days, new acute renal failure, need for dialysis or vasopressors) in patients with sepsis or septic shock. ${ }^{7}$ The trial was stopped early for safety reasons. ${ }^{7}$ Analyses of data for 537 patients showed that the rate of hypoglycemia was unacceptably high in the IIT group (17.0\% versus 4.1\%, ARI $12.9 \%$, NNH 8), ${ }^{7}$ and regression analysis indicated that hypoglycemia was an independent risk factor for death. ${ }^{7}$ Unrecognized adverse effects of hypoglycemia on the brain or heart might have offset the potential benefits of treatment.

Glucontrol was a multicentre randomized controlled trial involving 1078 medical and surgical ICU patients. The study compared a conventional blood glucose target of 7.8-10.0 $\mathrm{mmol} / \mathrm{L}$ with an intensive target of 4.4-6.1 mmol/L. ${ }^{8}$ Although the trial was terminated early because of a high rate of unintended protocol violations, there was no ICU mortality benefit (IIT $17.2 \%$ versus control $15.3 \%, p=0.41$ ), and the IIT group experienced significantly higher rates of severe hypoglycemia (8.7\% versus $2.7 \%$, ARI $6.0 \%$, NNH 17$){ }^{8}$

The Normoglycaemia in Intensive Care Evaluation Survival Using Glucose Algorithm Regulation (NICE-SUGAR) study was a prospective, multicentre randomized controlled trial with 6104 medical and surgical ICU patients. This study compared a conventional blood glucose target of $\leq 10 \mathrm{mmol} / \mathrm{L}$ with an intensive target $(4.5-6 \mathrm{mmol} / \mathrm{L}){ }^{9}$ In contrast to the studies by Van den Berghe's group, ${ }^{5,6}$ enteral nutrition was the primary source of nutrition, in accordance with current recommendations, which removed a potential confounder. ${ }^{15-17}$ There was no significant difference in 28-day mortality (IIT $22.3 \%$ versus $20.8 \%$ control, $p=0.17$ ), but the IIT group had significantly higher rates of severe hypoglycemia $(6.8 \%$ versus $0.5 \%$, ARI $6.3 \%$, NNH 16). ${ }^{9}$ Of most concern was the 90-day mortality rate, which was significantly higher in the IIT group than in the control group (27.5\% versus $24.9 \%$, ARI $2.6 \%$, NNH 38). ${ }^{9}$ The 
extended follow-up and much larger sample size of the NICESUGAR study provided sufficient power to detect a clinically important difference in mortality, which previous studies had not been able to do.

Clinicians should always abide by the ethical principle of "First, do no harm." Although the 2 studies by Van den Berghe's group appeared to show benefits with tight glucose control, 5,6 3 subsequent randomized controlled trials highlighted the harm associated with this approach, particularly increased mortality rates. $^{7-9}$ Although it is prudent to treat hyperglycemia (blood glucose $>10 \mathrm{mmol} / \mathrm{L}$ ) to prevent potential adverse outcomes, tight glycemic control does not reduce mortality; instead, it increases the risk of severe hypoglycemia over that of more liberal targets $(7.8-10 \mathrm{mmol} / \mathrm{L})$, as shown by the Glucontrol ${ }^{8}$ and NICE-SUGAR 9 studies. Weighing the risks and benefits of tight glucose control, we conclude that, until more convincing data become available, tight glycemic control should not be used. Rather, targeting blood glucose under $10 \mathrm{mmol} / \mathrm{L}$ is achievable and safe for critically ill patients.

\section{References}

1. Krinsley JS. Association between hyperglycemia and increased hospital mortality in a heterogenous population of critically ill patients. Mayo Clin Proc 2003;78(12):1471-1478.

2. Bochicchio GV, Sung J, Joshi M, Bochicchio K, Johnson SB, Meyer W, et al. Persistent hyperglycemia is predictive of outcome in critically ill trauma patients. J Trauma 2005;58(5):921-924.

3. Rovlias A, Kotsou S. The influence of hyperglycemia on neurological outcome in patients with severe head injury. Neurosurgery 2000; 46(2):335-342.

4. Leonidou L, Mouzaki A, Michalaki M, DeLastic AL, Kyriazopoulou V, Bassaris HP, et al. Cytokine production and hospital mortality in patients with sepsis-induced stress hyperglycemia. J Infect 2007; 55(4):340-346.

5. Van den Berghe G, Wouters P, Weekers F, Verwaest C, Bruyninckx F, Schetz $\mathrm{M}$, et al. Intensive insulin therapy in the critically ill patients. $N$ Engl J Med 2001;345(19):1359-1367.

6. Van den Berghe G, Wilmer A, Hermans G, Meersseman W, Wouters PJ, Milants I, et al. Intensive insulin therapy in the medical ICU. $N$ Engl J Med 2006;354(5):449-461.

7. Brunkhorst FM, Engel C, Bloos F, Meier-Hellman A, Ragaller M, Weiler $\mathrm{N}$, et al. Intensive insulin therapy and pentastarch resuscitation in severe sepsis. N Engl J Med 2008;358(2):125-139.
8. Preiser JC, Devos P, Ruiz-Santana S, Mélot C, Annane D, Groeneveld J, et al. A prospective randomised multi-centre controlled trial on tight glucose control by intensive insulin therapy in adult intensive care units: the Glucontrol study. Intensive Care Med 2009;35(10):1738-1748.

9. NICE-SUGAR Study Investigators; Finfer S, Chittock DR, Su SY, Blair $\mathrm{D}$, Foster $\mathrm{D}$, Dhingra $\mathrm{V}$, et al. Intensive versus conventional glucose control in critically ill patients. N Engl J Med 2009;360(13):1283-1297.

10. Vriesendorp TM, De Vries JH, Hoekstra JBL. Hypoglycemia and strict glycemic control in critically ill patients. Curr Opin Crit Care 2008; 14(4):397-402

11. Bord JG, Graber AL, Christman JW, Powers AC. Practical management of diabetes in critically ill patients. Am J Respir Crit Care Med 2001; 164(10):1763-1767.

12. Bassler D, Ferreira-Gonzalez I, Briel M, Cook D, Devereaux P, HeelsAnsdell D, et al. Sytematic reviewers neglect bias that result from trials stopped early for benefit. J Clin Epidemiol 2007;60(9):869-873.

13. Bassler D, Montori VM, Briel M, Glasziou P, Guyatt G. Early stopping of randomized clinical trials for over efficacy is problematic. J Clin Epidemiol 2008;61(3):241-246.

14. Sydes MR, Palmer M. Interim monitoring of efficacy data is important and appropriate. J Clin Epidemiol 2008;61(3):203-204.

15. Jolliet P, Pichard C, Biolo G, Chiloéro R, Grimble G, Leverve X, et al.; for the European Society of Intensive Care Medicine (ESICM) Working Group on Nutrition and Metabolism. Enteral nutrition in intensive care patients: a practical approach. Intensive Care Med 1998;24(8):848-859.

16. Heyland DK, Dhaliwal R, Drover JW, Gramlich L, Dodek P; Canadian Critical Care Clinical Practice Guidelines Committee. Canadian clinical practice guidelines for nutrition support in mechanically ventilated, critically ill adult patients. JPEN J Parenter Enter Nutr 2003;27(5):355-373.

17. Kreymann KG, Berger MM, Deutz NE, Hiesmayr M, Jolliet P, Kazandjiev G, et al. ESPEN guidelines on enteral nutrition: intensive care. Clin Nutr 2006;25(2):210-223.

18. Merz TM, Finfer S. Pro/con debate: Is intensive insulin therapy targeting tight blood glucose control of benefit in critically ill patients? Crit Care 2008;12(2):212.

19. Kanji S, Buffie J, Hutton B, Bunting PS, Singh A, McDonald K, et al. Reliablity of point-of-care testing for glucose measurement in critically ill adults. Crit Care Med 2005;33(12):2778-2785.

\section{Miranda So, BScPhm}

Silvia Duong, BScPhm

PharmD students

Leslie Dan Faculty of Pharmacy

University of Toronto

Toronto, Ontario 